\title{
Paysages : le ciel, la terre et l'eau
}

\section{Jacques Fontanille}

\section{OpenEdition}

Journals

Édition électronique

URL : http://journals.openedition.org/edl/503

DOI : $10.4000 /$ edl. 503

ISSN : 2296-5084

\section{Éditeur}

Université de Lausanne

\section{Édition imprimée}

Date de publication : 15 mai 2013

Pagination : 231-246

ISBN : 978-2-940331-32-1

ISSN : 0014-2026

\section{Référence électronique}

Jacques Fontanille, «Paysages : le ciel, la terre et l'eau », Études de lettres [En ligne], 1-2 | 2013, mis en ligne le 15 mai 2016, consulté le 18 décembre 2020. URL : http://journals.openedition.org/edl/503 ; DOI : https://doi.org/10.4000/edl.503 


\section{PAYSAGES : LE CIEL, LA TERRE ET L'EAU}

La définition sémiotique du paysage se joue dans la possibilité d'établir le rapport entre un espace figuratif, tributaire de la perception du sujet qui l'observe (plan de l'expression), et les valeurs qui en émanent (plan du contenu). Dans cette "sémiotique-objet", l'énonciation du paysage est l'acte par lequel expérience et existence se rencontrent pour signifier ensemble. Un paysage, dans notre héritage culturel occidental, a les propriétés d'une physionomie singulière. L'énonciation du paysage est donc aussi un moment d'iconisation et de singularisation où la résolution de toutes les hétérogénéités constitutives aboutit à la reconnaissance d'une identité figurative. Face à un paysage, le regard parcourt toujours à la fois un espace et une durée. Dans une seule énonciation, le paysage conjugue donc deux parcours narratifs: le récit de la morphologie dynamique du paysage, et celui de l'expérience sensible. Cet encadrement théorique du paysage-objet est vérifié, à titre d'exemple, à travers l'analyse de la construction iconique du paysage limousin tel qu'il est mobilisé par un échantillon de photographies représentatives.

\section{Le paysage comme "sémiotique-objet»}

D'un point de vue sémiotique, un paysage se présente d'abord comme un segment du monde naturel, un espace figuratif, dont la valeur émane des sensations et perceptions qu'il procure, eu égard à une position d'observation. Le rapport entre les propriétés sensibles de cet espace figuratif (le plan de l'expression), et les valeurs qui en émanent (le plan du contenu) est la fonction sémiotique que le sémioticien se propose d'établir. Néanmoins, il faut ajouter deux conditions pour que cette fonction sémiotique soit opérante: un rapport à l'histoire et un rapport au territoire.

En effet, un paysage privé de toute dimension temporelle n'aurait aucune valeur. Certes, le paysage-espace ne manque pas de contrastes, 
mais ils ne valent qu'en raison de leur caractère dynamique, c'est-à-dire de ce qu'ils ont été auparavant, et de ce qu'ils deviendront bientôt. En outre, un paysage n'en reste pas moins une partie d'un territoire: toutes les propriétés des parties environnantes continuent à agir de manière latente sur la perception et l'interprétation du segment de paysage retenu. Le rapport au territoire, tout comme le rapport à l'histoire, est constitutif du sens du paysage.

\section{Remarques sur les valeurs du paysage}

2.1. Les deux sens de "valeur» en sémiotique

Attribuer ou reconnaître une valeur dans une sémiotique-objet, c'est d'abord identifier des différences. Attribuer de la valeur, c'est aussi, ensuite, polariser ces figures différenciées, les orienter, positivement ou négativement, les rendre désirables ou utiles, nécessaires ou admirables (ou le contraire!). Ce sont ces deux propriétés, la différence et la polarité, qui constituent des valeurs sémiotiques et que nous retrouvons dans le paysage.

Les valeurs-différences du paysage sont de deux sortes: des différences sensibles, perceptibles, saisissables dans l'expérience que nous procure le paysage; et des différences existentielles, géologiques, historiques, économiques, que l'on peut constituer en "plan du contenu». Des valeurs différentielles relevant respectivement de l'expérience et de l'existence s'associent alors pour constituer la relation sémiotique élémentaire entre un plan de l'expression et un plan du contenu. L'énonciation du paysage est l'acte par lequel «expérience» et "existence», différences sensibles et différences existentielles, se rencontrent pour signifier ensemble: cet acte impersonnel est un «effet de site».

Quant aux objets de valeur que l'on recherche en tant que contenus, ils appartiennent aux usages et aux pratiques auxquelles le paysage invite et qu'il propose sous forme de promesse adressée à l'observateur: le paysage est à cet égard un condensé de souvenirs et de promesses: promesses et souvenirs perceptifs, promesses et souvenirs pratiques, par lesquels il invite à la contemplation, au parcours, à la visite, au séjour ou à toute autre activité. Dès lors, les "valeurs différentielles", telles qu'elles sont saisies par l'observateur, sont prises en charge dans la 
perspective temporelle et syntagmatique d'une manipulation cognitive et passionnelle de l'observateur-participant, sous forme de "promesses", «incitations», "invitation», "suggestion», "proposition», etc., dans le récit en puissance du paysage saisi comme instantané dynamique.

\subsection{Le moment iconique}

Le paysage peut donc être appréhendé sous deux dimensions complémentaires: la dimension de l'existence, composée de "thématiques» disparates (géologique, morphologique, économique, historique, etc.), et la dimension de l'expérience, constituée d'un ensemble de phénomènes hétérogènes et de propriétés sensibles diverses. Cette hétérogénéité appelle une résolution et même, dans le cas du paysage, une forme d'identité: un paysage, dans notre héritage culturel occidental, a les propriétés d'une "physionomie» singulière; on l'identifie, on le reconnaît; il a un "type» et des traits individuels. L'énonciation du paysage est donc aussi un moment d'iconisation et de singularisation, où la résolution de toutes les hétérogénéités constitutives aboutit à la reconnaissance d'une identité figurative.

La composante méréologique et topologique

Un paysage est un assemblage de parties-objets, réglé par une perception: c'en est même la définition juridique. Mais la perception et le point de vue ne font que reconnaître des alliances, des superpositions, des contiguïtés constatées ou possibles. Et, en outre, la "physionomie» et le moment iconique ne sont accessibles que si on peut identifier un principe organisateur interne: une rivière qui tranche, sépare et/ou connecte les autres parties, par exemple; ou un échange de formes, de textures et de reflets entre le ciel et un lac. Par conséquent, le «moment iconique» du paysage comporte une détermination topologique, plus ou moins dépendante ou indépendante du point de vue adopté.

\section{La composante temporelle}

Le point de vue qui constitue le paysage n'est jamais fixe: le panorama implique un balayage visuel et sa découverte dynamique implique une progression et une mobilité corporelle. La détermination temporelle concerne d'abord le réglage du point de vue. Mais la morphologie du 
paysage lui-même se donne toujours à saisir comme un ensemble de produits temporels de l'histoire qui sont distribués et reconnaissables dans des formes spatiales.

Du côté de l'expérience sensible, des propriétés comme la distance ou la profondeur, à la différence de ce qui se passe face à un tableau, sont toujours à parcourir, et à pénétrer: face à un paysage, le regard parcourt toujours à la fois un espace et une durée. Du côté de l'existence du paysage, on doit prendre en compte le fait que c'est la morphologie même qui est soumise au temps, et la reconnaissance momentanée des formes du paysage n'introduit aucune discontinuité dans le processus temporel: elle permet justement d'appréhender le mouvement en cours et les valeurs impliquées dans ces processus temporels.

\section{La composante narrative}

Le paysage conjugue deux parcours narratifs qui exploitent les deux régimes temporels évoqués, en une seule énonciation: le récit de la morphologie dynamique du paysage, et celui de l'expérience sensible.

La confrontation entre ces deux récits est décisive pour la compréhension du paysage en tant qu'ensemble signifiant. Le récit existentiel procure au paysage un certain nombre de propriétés qui le rendent plus ou moins adapté à telle ou telle pratique: ce sont les "promesses et souvenirs» évoqués plus haut. Et il faut donc que l'autre récit, le récit de l'expérience, puisse se déployer parmi ces promesses et souvenirs. Du point de vue narratif, en somme, le paysage canonique est celui que nous appréhendons au cours d'une pratique qui est à la fois adaptée à la morphologie du lieu et propice au déploiement de l'expérience sensible. Mais cette conjoncture idéale est affectée de nombreuses variations et l'ajustement entre les deux régimes est bien souvent aléatoire, compromis, et irrégulier: c'est alors qu'on peut faire l'expérience de la résistance du paysage.

\section{Paysages limousins: le récit d'une capture mythique}

\subsection{La constitution du corpus}

En matière de corpus de paysages, comme pour toutes les sémiotiques du monde naturel, l'exhaustivité est hors de portée: les paysages ne 
constituent en effet pas des corpus mobilisables et manipulables dans les conditions habituelles de la recherche. Il faut donc procéder à des extractions successives, en rapport avec les objectifs poursuivis, et qui visent une représentativité limitée. Ici-même, et pour obtenir un corpus mobilisable et analysable, on acceptera provisoirement une réduction à la dimension visuelle du paysage, à quelques photographies où l'on perd certes la plupart des données d'expérience, mais qui permettent au moins d'isoler la dimension plastique de l'énonciation, et d'extraire tout particulièrement le rôle de la lumière et de l'atmosphère dans la sémiose.

Le choix de l'échantillon repose sur la sélection d'un certain nombre de propriétés pertinentes et représentatives d'un territoire:

(1) les composants figuratifs (terre, air, eau, acteurs, bâti, formes spécifiques, etc.);

(2) les propriétés sensibles et les contrastes de la dimension plastique;

(3) les distances et les positions d'observation;

(4) les accidents figuratifs.

3.2. Paysages de collines et de plateaux: la lumière, l'atmosphère et l'horizon (pl. I.2)

La construction en trois plans (premier/second/arrière-plan) repose ici sur les variations de saturation d'une même gamme chromatique, accompagnées de variations de netteté des formes: le vert saturé sombre, devient délavé, flou et brumeux à la limite de l'horizon. Ces propriétés plastiques suggèrent, du côté du contenu, la présence d'une épaisseur invisible qui fait écran (l'atmosphère) et qui modifie la réaction des différents plans de profondeur à la lumière: en somme, il y a un contrôle qui capture et absorbe une partie de la lumière, et de plus en plus quand on s'enfonce en profondeur.

La composition chromatique a donc pour corrélat des valeurs d'interaction entre les matières et la lumière, sous le contrôle de l'atmosphère: les masses vert sombre, dont la texture absorbe la lumière, s'opposent aux surfaces vert désaturé et clair qui, certes, captent la lumière, mais la restituent. Cette composition se présente d'abord sous sa dimension plastique, où les propriétés de traitement de la lumière - textures, structures matérielles, couleur - permettent de distinguer une composition figurative. Mais on constate que le contrôle de l'atmosphère tend à "défigurer " le paysage: quand l'épaisseur atmosphérique, au premier plan, est 
réduite, l'action est faible et les figures sont aisément reconnaissables, car leur couleur, leur texture et leurs formes sont bien contrastées; en revanche, quand l'épaisseur atmosphérique est maximale, à l'arrière-plan, la défiguration est forte, et les formes sont à peine reconnaissables, les contrastes plastiques étant atténués.

L'ensemble de ces remarques conduit alors à s'interroger sur le rapport avec le ciel. La ligne d'horizon est à la fois une ligne de rupture, une frontière, et une zone de transition inverse. Sur le plan chromatique, les tons sont différents (dominante de verts-bruns dans un cas, dominante de bleus dans l'autre), et les deux zones s'opposent comme deux ensembles figuratifs distincts; et pourtant, à l'horizon, les deux ensembles, terre et végétaux d'un côté, ciel de l'autre, connaissent la même désaturation, de sorte que leur différence s'amenuise. Gardons en réserve cette observation, qui sera complétée par d'autres bientôt.

Ces deux vues (pls II.1 et 2) exploitent les mêmes types définis plus haut, puisqu'on y retrouve:

- les principes de composition chromatique en plans de profondeur;

- le principe de composition plastique en contrastes systématiques et dégressifs.

Mais s'ajoute ici un fort contraste de distance. En II.2, la distance plus rapprochée permet:

- de distinguer des objets et pas seulement des masses (les fleurs, les barrières dans le pré, etc.);

- de reconnaître la forme spécifique d'une pente et l'arrondi d'une colline (dans I.2, ces formes spécifiques du lieu étaient neutralisées par la distance et la superposition des plans);

- de disposer les plans de profondeur dans la hauteur de la vue, et pas seulement dans l'épaisseur des masses d'air.

En II.2, la distance est maximale, mais cette fois pourtant elle ne neutralise pas les formes, car les formes spécifiques (les massifs montagneux) étant de taille beaucoup plus importante, elles ne sont pas sensibles à la distance d'observation et on reconnaît la forme de sommets volcaniques. Les effets de taille et de distance sont ici fortement contraints par la morphologie du paysage, et la distance d'observation n'a que peu de prise sur ses propriétés sensibles. Pourtant, l'atmosphère agit toujours aussi efficacement: la gamme des "désaturations" et des "détexturations", et l'échelle des épaisseurs «brumeuses» manifestent tout aussi clairement les plans de profondeur; cette action apparaît donc ici sans conteste 
comme indépendante de l'observateur et propre au contrôle interne du paysage (l'atmosphère). Elle est donc «objective».

La confrontation en série des trois premiers paysages permet maintenant de préciser le rapport avec le ciel: cette série met en effet en évidence, autour de la ligne d'horizon, un rapport d'inversion des gammes de désaturation et de détexturation. Dans la zone terrestre comme dans la zone céleste, plus on s'approche de cette frontière, plus les tons sont désaturés et plus les surfaces sont détexturées; au fur et à mesure qu'on s'éloigne de cette ligne, les tons retrouvent leur vivacité et leur éclat, tandis que les surfaces et les masses retrouvent leurs textures et leurs formes.

Par hypothèse, on pourrait suggérer que l'horizon n'appartenant à aucune des deux zones, ni au ciel ni à la terre, il est l'axe de symétrie de ces deux zones, symétrie des propriétés plastiques de saturation/ désaturation et de texturation/détexturation; il est la limite où l'ensemble «terre et végétaux» et l'ensemble "ciel» pourraient se confondre, s'ils n'étaient pas de couleurs différentes. Mais même cette différence tend dans certains cas à s'estomper; on voit apparaître des nuances de tons mêlés (des gris tirant sur le bleu), et entièrement désaturés (II.2) tout près de la limite. Cette limite est donc celle de l'inversion des processus de saturation/texturation, tout autant que celle entre deux espaces figuratifs.

C'est alors qu'il faut se rappeler l'activité de contrôle dans la zone terrestre: l'atmosphère modifie les couleurs, les textures et les formes de la terre et des végétaux, en fonction de la profondeur (et de sa propre épaisseur), en modulant l'action de la lumière. A propos du ciel, un tel raisonnement ne peut être reconduit, puisque c'est justement l'atmosphère elle-même que l'on perçoit comme "ciel», et il n'y a rien d'autre à modifier que de l'atmosphère.

Il faut alors réinterpréter cette articulation majeure de tout paysage comme un rapport d'englobement et de détermination unilatérale: l'atmosphère et la lumière sont en quelque sorte les deux acteurs principaux de cette mise en scène phénoménologique; la lumière agit sur des figures, et l'atmosphère agit sur la manière dont la lumière agit sur les figures. Dès lors, la différence entre les deux ensembles figuratifs tient au fait que dans la zone terrestre ces actions sont transitives (elles s'appliquent aux masses terrestres et végétales, c'est-à-dire à autre chose que l'atmosphère et la lumière), alors que dans le second, ces actions sont 
réflexives (l'atmosphère et la lumière s'appliquent à elles-mêmes les jeux de la profondeur et de la distance).

Mais, de ce fait même, l'activité de la lumière (opérateur) et de l'atmosphère (contrôle) constitue un facteur d'englobement absolu de tout paysage (et pas seulement limousin), et elle est susceptible de produire deux effets:

- soit l'effet "profondeur" quand elle a pour objet les masses terrestres et végétales, dans une action transitive;

- soit l'effet «ciel» quand elle se prend elle-même pour objet, dans une action réflexive.

Ce rapport d'englobement est donc techniquement supporté par une opération énonciative, qui est:

- soit de débrayage, quand l'activité est transitive (la lumière et l'atmosphère mettent en scène autre chose qu'elles-mêmes);

- soit d'embrayage, quand l'activité est réflexive (elles ne parlent que d'elles-mêmes).

L'instance d'énonciation dans un paysage quelconque est donc constituée a minima de deux acteurs (lumière et atmosphère), qui agissent sur les propriétés plastiques des figures qui le composent. Cette instance est invisible et implicite dans les parties terrestres sur lesquelles elle agit "transitivement", alors qu'elle est visible, embrayée et représentée, dans le ciel, où elle agit "réflexivement» sur elle-même. Pour faire bref, le ciel serait la représentation, inscrite dans le paysage même, du foyer d'énonciation, d'une instance d'énonciation qui s'énonce elle-même en se «rendant visible». De ce fait, la ligne d'horizon matérialise en somme la frontière entre les opérations de débrayage et d'embrayage: débrayage en deçà, embrayage au-delà.

On retrouve ici (pl. II.3) les principes reconnus précédemment, mais sous quelques conditions particulières qui perturbent à la fois l'appréciation de la distance, et l'interaction entre la zone céleste et la zone terrestre. Ce sont: (1) l'affaiblissement du rôle de l'atmosphère dans le contrôle de la profondeur, (2) l'extension disproportionnée du second plan (le plan principal) ainsi que la réduction a minima du premier plan et de l'arrière-plan et (3) la réduction des contrastes cohérents à une opposition entre une seule masse claire et brune, et une multitude de masses vert foncé disposées en périphérie.

Il en résulte que le spectateur n'est plus en mesure d'apprécier la taille et la profondeur de la masse claire, et aussi la taille respective des différentes masses végétales (notamment celle des arbres isolés). En outre, 
et surtout, le contraste principal, organisateur du paysage, se produit entre la grande plage brun clair et la grande plage de ciel bleu. On peut alors déceler et poser en hypothèse l'existence d'une tension structurante entre d'un côté la dimension figurative et de l'autre la dimension plastique, cette tension étant réglée par l'appréciation des profondeurs en fonction de la distance du point de vue et de l'action de l'atmosphère.

Dans certaines configurations "canoniques", la morphologie plastique du lieu et la distance de vue entrent en résonance, et concourent à la reconnaissance de la composition figurative. Dans d'autres configurations, les deux dimensions se combattent plus ou moins, entraînant quelques difficultés de «recollement» entre la composition figurative, le positionnement du point de vue et les rimes et équivalences plastiques.

Dans le cas de cette dernière vue, cette difficulté de recollement favorise les équivalences plastiques, et notamment le dialogue direct et paradoxal entre le ciel et une des grandes plages terrestres. On peut alors observer que cette plage terrestre ne se caractérise pas seulement par sa taille et son caractère central, mais aussi par sa désaturation, qui fait écho à celle du ciel, dans un autre chromatisme.

Il y a donc d'autres solutions possibles, pour l'articulation entre le ciel et la terre, que la ligne d'horizon: les mêmes relations peuvent advenir dans un rapport haut/bas, entre des zones parallèles où l'inversion fonctionne en miroir et non en symétrie. C'est d'une de ces constructions non canoniques que nous voudrions maintenant rendre compte, avec les paysages lacustres.

\subsection{Paysages lacustres: la délégation céleste (pls II.4 et 5)}

La comparaison avec les paysages de terre et de collines montre que toutes les catégories précédentes s'appliquent de la même manière, alors que le lac ou l'étang, au milieu des terres, des prairies et des collines, est un élément obéissant en tous points à d'autres catégories:

- la régularité de la surface s'oppose à l'irrégularité des terres et des ensembles boisés;

- la planéité horizontale s'oppose à la rotondité, à l'oblicité, à la sinuosité des surfaces;

- les variétés de bleu s'opposent aux variétés de bruns et de vert.

Le lac n'entretient donc de relations plausibles qu'avec le ciel et non avec la terre et la verdure, puisque c'est seulement avec le ciel qu'il partage 
la plupart de ses propriétés plastiques et qu'il peut entretenir un dialogue signifiant. Les couleurs et les textures de ces deux ensembles, notamment, varient de manière congruente, de sorte que, même si le lac et le ciel présentent des nuances chromatiques différentes, elles sont imputables à la différence de matière et de position (eau/air; bas/haut; proche/ lointain), et elles n'affectent pas l'«écho" chromatique et la variation corrélée des deux ensembles, à l'intérieur d'une série.

Cette variation, attribuable aux aléas de la lumière, conforte la distinction entre deux ensembles figuratifs:

- le premier, celui des terres et de la végétation, dont on a vu que les dégradés de saturation dépendent aussi de la lumière et de la distance, - le second, celui des lacs et des ciels, qui réagissent eux aussi à la lumière, mais avec un chromatisme et une texture qui les distinguent radicalement des premiers.

Témoigne de ce rôle essentiel de la lumière le fait qu'à l'intérieur des paysages de lacs et de ciels, la différence de matière s'exprime essentiellement par une différence de traitement de la lumière: reflets moirés d'un côté, transparence diaphane de l'autre. Mais les deux suscitent pourtant également un effet de surface, qui s'oppose globalement aux effets de masses et de textures du premier ensemble.

Comme par ailleurs les lacs et les étangs constituent le point focal de cette catégorie de paysages, puisqu'ils sont toujours "entre" les terres, entre les versants, ils ont pour rôle de les "agréger" " ${ }^{1}$ Or, le ciel, qui présente les mêmes propriétés plastiques, ne peut pas jouer ce rôle entre les parties du paysage, car il est un «englobant», alors que les lacs et les étangs sont des "englobés".

Il y a donc quelque chose de mythique, au sens technique de LéviStrauss, dans cette opération de construction: on peut en effet considérer que le ciel, qui présente toutes les propriétés pour agréger entre elles toutes les parties du paysage, et qui ne le peut pas en raison de sa position englobante, délègue au sein du paysage une figure englobée, avec laquelle il dialogue et varie de manière synchrone et qui, elle, peut jouer le rôle de liant et constituer le paysage en «agrégat». Le lac est donc

I. Ils obéissent à la définition technique de l'«agrégat", en méréologie, car ils constituent le lien principal, dans des ensembles dont ils sont la seule partie d'un genre différent de toutes les autres, et pourtant en contact avec toutes les autres. 
la figure de médiation entre le ciel et les terres, médiation supportée par une opération de débrayage.

L'hypothèse formée plus haut se confirme dès qu'on reconnaît, grâce à des vues plus rapprochées, des reflets et des ombres du paysage sur la surface du lac ou de l'étang (pls III.1 et 2). Ces reflets et ces ombres, en effet, confirment le statut très particulier de ces surfaces qui renvoient la lumière et les images, les captent pour les restituer au spectateur, mais inversées; ils mettent aussi en évidence leur rôle de contact et d'agrégation des autres parties du paysage, dont elles "accueillent» et entremêlent les images.

Le «moment iconique» du paysage se présente ici comme un «nœud» morphologique: d'un côté, le ciel et l'eau sont affectés de variations synchrones, grâce auxquelles ils dialoguent entre eux, et de l'autre, l'eau reflète le ciel, en même temps que les autres parties du paysage.

Le lac est un opérateur sémiotique qui produit de la mimésis: il capte les propriétés plastiques de toutes les parties du paysage, y compris le ciel, et les fait siennes; il est susceptible de "se faire" colline, arbre, falaise, tout comme ciel ou nuage; il capte aussi bien les propriétés des parties englobées que celles de l'atmosphère englobante, et, grâce à son pouvoir réfléchissant, le ciel englobant devient un reflet englobé parmi les reflets des autres parties du paysage: la rétroaction est complète, et le lac est bien le moyen (mythique) par lequel l'extériorité du ciel, par rapport aux terres et à la végétation, est neutralisée, grâce à cette projection commune de leurs images reflétées.

Il nous faut revenir ici aux opérations énonciatives évoquées plus haut, ainsi qu'à l'activité de la lumière et de l'atmosphère. Pour commencer, ce que nous avons appelé "délégation" et "dialogue», par facilité d'expression, n'est autre qu'une des formes du «débrayage» évoqué plus haut. Mais il s'agit d'un débrayage très particulier, qui consiste à mettre en scène non pas une altérité radicale (les masses terrestres et végétales), ni même une identité (le ciel en tant que tel), mais un véritable alter ego, un "autre soi-même» (le reflet du ciel).

Le débrayage que nous évoquions plus haut rend possible la mise en scène d'autres entités que celles qui ont en charge l'énonciation. De même, l'embrayage que nous avons reconnu dans la mise en scène réflexive du ciel permet une représentation dans le paysage même de son propre foyer d'énonciation. Mais le débrayage qui fait de la surface lacustre un alter ego du ciel délègue dans le paysage un autre foyer 
d'énonciation, une énonciation déléguée qui mettra elle-même en scène d'autres parties du paysage. Et grâce aux reflets, le ciel lui-même vient s'installer au cœur du nouveau dispositif.

La ligne d'horizon et le reflet sur les surfaces lacustres jouent donc le même type de rôle (des opérateurs figuratifs de débrayage et d'embrayage) mais sur des registres différents: la ligne d'horizon opère sur la présence et l'absence du foyer d'énonciation dans le paysage visible, alors que le reflet aquatique permet la duplication ou la multiplication des foyers d'énonciation délégués, au sein même du paysage terrestre.

\subsection{Paysages de rivières: la capture et l'absorption (pl. III.3)}

Alors que le lac s'insère entre les collines et les massifs végétaux, la rivière tranche, trace, sépare: elle joue de fait plus radicalement encore un rôle d'agrégation des parties, puisque sa présence et son passage suffisent à expliquer de manière convergente la forme et la présence de toutes les parties adjacentes qu'elle associe. En outre, la rivière offre elle aussi une surface, tout comme le lac, mais cette surface reçoit en outre une texture propre, voire plusieurs types de structures, selon la pente ou la nature du fond ou des berges.

Autre différence avec le lac (pls III.4 et 5): l'intimité entre la rivière et les parties qu'elle sépare et assemble en même temps est telle que son cours est encombré par des éléments de terre (des rochers) ou de végétation (des troncs et des branches). La réunion des deux ensembles ne repose donc plus seulement sur la conjonction des reflets et des ombres, mais sur la conjonction d'éléments appartenant aux parties adjacentes et capturés par la rivière; comme par ailleurs certaines surfaces de rivières peuvent aussi refléter les berges, les deux formes de "capture» co-habitent, se confrontent, et une séquence progressive se dessine, où la capture des reflets et des ombres est suivie de la capture des objets figuratifs eux-mêmes.

La différence suivante est chromatique (pl. IV.1) : la rivière a sa propre couleur, elle ne la reçoit donc pas du ciel. On sait par ailleurs que cette couleur dépend de la nature des sols et des alluvions et de fait, ayant "capturé» certaines des propriétés des terres et des végétaux, elle participe plus aux contrastes des verts et des bruns, propres à cet ensemble figuratif, et moins à ceux de l'ensemble "ciels et lacs». La rivière "épouse» la matière, les formes et les accidents des parties du paysage qu'elle est supposée associer entre elles; dans ce cas (cascade, pierrée ou 
chute), elle n'est plus qu'un habillage de texture et de couleur différentes des pentes ou des rochers; elle n'est plus en somme qu'un résidu de ciel capturé et prisonnier des formes et des matières terrestres.

Le débrayage est maintenant irréversible et on assiste à une absorption complète des figures aquatiques dans les masses et formes terrestres et végétales. Le ciel n'a plus d'alter ego, ne contrôle plus les surfaces aquatiques déléguées, la terre a vaincu et s'auto-organise.

Le ciel est finalement renvoyé à son altérité et même à son extériorité. A la limite, dans certaines approches de la rivière, il n'y a plus ou presque plus de ciel et la présence du foyer d'énonciation est alors entièrement virtuelle, et invisible.

\section{Conclusion}

Les valeurs du paysage sont bien sûr les valeurs attachées aux pratiques qu'on peut réaliser en lui, avec lui, ou entouré par lui. Mais ces valeurs pratiques et d'usage en présupposent d'autres qui les préparent et les rendent possibles, et qui sont tout simplement les valeurs perçues, les valeurs telles qu'elles se donnent à saisir dans le rapport sensible avec le paysage.

Et si on «remonte» au tout début du processus d'engendrement du sens, la première question qui se pose est celle de la signification des propriétés plastiques, ces propriétés qui se donnent à nous dans leur évidence première, avant même que nous ayons pu reconnaître quelque figure paysagère que ce soit, quelque type canonique que ce soit.

Nous l'avons vu, les propriétés plastiques donnent accès ici à un récit mythique élémentaire, dont les étapes et les acteurs forment l'armature sémiotique de tous les paysages naturels d'un même territoire. Des acteurs paysagers: l'opérateur "lumière», le contrôle "atmosphère", les objets que sont: végétaux, masses terrestres et rocheuses, espaces célestes, surfaces ou bandes d'eau, etc.

Il faut également mettre en œuvre des opérations énonciatives de débrayage et d'embrayage qui permettent de manifester soit les «objets» figuratifs (en profondeur), soit le foyer d'énonciation lui-même (lumière et atmosphère $=$ ciel), et qui peuvent également déléguer ces mêmes opérations à des surfaces aquatiques intimement associées aux autres parties du paysage. Ces opérations produisent des médiations figuratives, porteuses de la dynamique du paysage-récit. 
Ce dispositif élémentaire donne lieu à quelques événements sémiotiques dont nous n'avons exploré qu'une infime partie, mais autour de quelques échantillons très suggestifs; ainsi avons-nous assisté à l'invention de la profondeur, et à son articulation symétrique avec le ciel; à la capture des images du paysage et du ciel par les surfaces lacustres; et enfin à l'absorption des restes de ciel, à travers les eaux courantes des rivières, dans les masses et les formes terrestres. On voit bien ici se dessiner le théâtre des valeurs paysagères, avec ses jeux de rôles profonds, ses séquences canoniques et ses systèmes de valeurs.

Jacques Fontanille

Université de Limoges 


\section{Crédits iconographiques}

Pl. I.2 à IV.1 :

Service de cartographie de la Faculté des Lettres et Sciences Humaines de Limoges, Université de Limoges. 
